REgina GRIgor'Eva \& TATJANA LUKJANČENKO

\title{
Zur Erforschung moderner ethnokultureller Prozesse bei den Lappen der Halbinsel Kola
}

In den Jahren 1985-1986 haben wir eine statistisch-ethnographische Untersuchung der Lappen der Halbinsel Kola im Hinblick auf die gegenwärtig unter ihnen stattfindenden ethnokulturellen Prozesse durchgeführt. Ziel war unter anderem, die Gesetzmäßigkeiten und Tendenzen der ethnokulturellen Entwicklung der Lappen und die diese Entwicklung beeinflussenden Faktoren, aber auch die Art ihrer Kontakte zu anderen Nationen zu ermitteln. Die Untersuchung hing darüber hinaus mit der Notwendigkeit zusammen, mehrere praktische Aufgaben im sozialen und wirtschaftlichen Leben der Lappen zu lösen, was nur unter Berücksichtigung der ethnischen Besonderheiten des Volkes möglich ist. Aus diesem Grunde nahmen wir uns vor, das Niveau der Zweisprachigkeit der Lappen, deren Bedürfnis, ihre Kinder in der Schule die Muttersprache lernen zu lassen, die gegenseitige Verständlichkeit zwischen den verschiedenen Dialektgruppen, den Beschäftigungsgrad in den traditionellen Tätigkeitsarten zu ergründen, aber auch festzustellen, ob und in welchem Maß die traditionelle Pelzbekleidung immer noch Gebrauch findet, welche Nahrungsmittel bevorzugt werden $\mathrm{u}$. dgl. $\mathrm{m}$.

Aufgrund der konkreten Aufgaben haben wir einen Fragebogen mit 124 nach Themenkreisen geordneten Fragen zusammengestellt, über die Beschäftigungsarten der Bevölkerung, über das soziale und berufliche Vorankommen im Verlauf des Lebens, über die Familie, über die Kontakte zu anderen Nationen, über die 
sprachliche Situation, über die materielle Kultur, über die Riten usw. Zu jeder Frage wurde das jeweilige Verhältnis zwischen den traditionellen und den modernen Kulturelementen geklärt, wobei die Fragen innerhalb jedes Themenkreises so formuliert waren, daß die entsprechenden Antworten eine Vorstellung von dem Wissen der Befragten um die traditionellen Formen der Kultur vermittelten, aber auch von der Nutzung dieser Formen in der Gegenwart und von der Einstellung der Befragten zu diesen Formen, das heißt von der ethnischen Haltung der Menschen.

Nach der Volkszählung von 1979 leben auf der Halbinsel Kola bekanntlich 1900 Lappen, davon 1600, das heißt an 85 Prozent, im Gebiet Murmansk. Etwa 30 Prozent der Lappen leben in Städten und Siedlungen städtischen Typs. Das sind vor allem die Städte Murmansk, Močegorsk und Kirovsk und Siedlungen städtischen Typs wie Revda und Gremiha. Unter den in Städten lebenden Lappen sind fast 60 Prozent Frauen, die sonst 55 Prozent der lappischen Bevölkerung ausmachen. Unter den städtischen Lappen führen die meisten Mischehen mit Russen, Ukrainern, Kareliern und Vertretern bzw. Vertreterinnen anderer Nationalitäten.

Die restlichen 70 Prozent (das heißt an 1100 Personen) gehören zur ländlichen Bevölkerung. Die ländliche Bevölkerung der Lappen im Gebiet Murmansk ist durch drei territoriale Gruppen vertreten. Der Hauptteil der ländlichen Lappen (an die 900) lebt im Bezirk Lovozero, die meisten von ihnen in den Siedlungen Lovozero und Krasnoščel'e im Mittelpunkt der Halbinsel Kola. Sie sind sowohl in herkömmlichen Wirtschaftsbereichen wie Rentierzucht und Fischerei als auch in für sie neuen wie Milchviehhaltung, Ackerbau, Bauwesen, aber auch Bildungswesen und Dienstleistungsbereich beschäftigt.

Zwei andere kleine Gruppen leben entsprechend im Bezirk Kola im nordwestlichen Teil des Gebiets Murmansk, wo die meisten Lappen gemeinsam mit Repräsentanten anderer Völkerschaften im Viehsowchos „Tuloma“ arbeiten, und im Südwesten des Gebiets Murmansk im Dorf Ëna und dessen Umgebung. Diese Lappen sind ebenfalls in für sie nicht traditionellen Wirtschaftsbereichen beschäftigt. 
Wir haben die Lappen von Lovozero als die zahlenmäßig stärkste Gruppe unter allen zum Objekt unserer Untersuchung gemacht und uns dabei vorgenommen, letztere nach Möglichkeit durchgehend $\mathrm{zu}$ gestalten. Die Personalien der zu befragenden Menschen unter Angabe von Vor- und Nachnamen, Alter, Hausadresse und Arbeitsstelle haben wir den Hausbüchern entnommen, die es in jedem Dorfsowjet, so auch in dem von Lovozero und von Krasnoščel'e, gibt und die entsprechende Angaben über jede in der jeweiligen Siedlung wohnhafte Familie enthalten. Unsere Expedition und folglich auch die Befragung fand einmal im Sommer und einmal im Winter statt.

Im Ergebnis wurden in den beiden Siedlungen Lovozero und Krasnosčel'e 400 Lappen ab 16 Jahre interviewt, was etwa 70 Prozent der dort lebenden erwachsenen Lappen ausmachte. Die gesammelten Materialien konnten bis jetzt nur zum Teil ausgewertet werden. Man kann aber bereits von einigen, wenn auch vorläufigen Schlüssen sprechen. Im folgenden wollen wir auf zwei Fragen eingehen: die Kontakte der Lappen der Halbinsel Kola zu anderen Völkerschaften und die ethnolinguistische Situation unter ihnen.

Wie die Untersuchung der Lappen im Bezirk Lovozero ergab, leben in den Dörfern Lovozero und Krasnoščel'e 252 lappische Familien, darunter 222 in Lovozero und 30 in Krasnoščele. Diese beiden Siedlungen unterscheiden sich stark voneinander nach dem administrativen Status, der Einwohnerzahl, der nationalen Zusammensetzung, nach der sozialen und beruflichen Struktur u. dgl. m., was wiederum zwangsläufig seinen Niederschlag in der ethnokulturellen Entwicklung findet. Lovozero, ein angestammter Siedlungsort der Lappen, ist als solcher aus entsprechenden Quellen spätestens seit Anfang des 17. Jh. bekannt. In den 50er und 60er Jahren unseres Jahrhunderts, als im Gebiet Murmansk Dörfer vergrößert und in der Nähe einiger lappischer Pogoste Wasserkraftwerke gebaut wurden, siedelte ein beträchtlicher Teil der Lappen aus anderen kleinen Siedlungen wie Čudsjavr, Voron'e, Jokanga und Varzino nach Lovozero um. Auf diese Weise konzentrierte sich in Lovozero der Hauptteil der im heuti- 
gen Bezirk mit diesem Namen lebenden Lappen (etwa 85 Prozent). Dabei leben dort nun Vertreter verschiedener Dialekt- und Lokalmundartengruppen (Kildiner- und Jokangaer Dialekt, Lokalmundarten von Semiostrov'e, Voron'e u. a.). In Lovozero befindet sich der Rentierzucht-Sowchos „Tundra“, der größte im Gebiet Murmansk. Lovozero ist ferner die Hauptstadt eines Bezirks, in dem neben der Landwirtschaft (insbesondere Rentierzucht, Jagd und Fischfang) auch die Industrie entwickelt ist. Die Entwicklung der Industrie führt unter anderem dazu, da $\beta$ im Bezirk eine ständige Zuwanderung beobachtet wird, wobei die Menschen zum Teil auch in Lovozero seßhaft werden.

Lovozero hat eine gemischte nationale Zusammensetzung. Mehr als die Hälfte seiner Einwohner sind Russen und Zugewanderte anderer Nationalitäten; knapp je ein Viertel sind Lappen und Syrjänen; ein kleiner Rest von rund 2,5 Prozent sind Nenzen.

Krasnoščel'e, eine verhältnismäßig junge Siedlung, wurde 1921 von syrjänischen Rentierzüchtern am Fluß Ponoj gegründet. $\mathrm{Zu}$ Lovozero und anderen Orten des Gebiets besteht nur eine Flugverbindung. Die Syrjänen stellen von Anfang an das Gros der Einwohner (zur Zeit über die Hälfte). Bis Mitte der 60er Jahre lebten so gut wie überhaupt keine Lappen in Krasnoščele. Erst um diese Zeit siedelten sie aus dem ein Stück weiter unter am Ponoj gelegenen Dorf Ivanovka, weil dort der Bau eines Staudamms geplant wurde, nach Krasnoščele um. Es sind Lappen aus dem ehemaligen Pogost Kamenskij, Vertreter der Jokanger Dialektgruppe. In Krasnoščele machen die Lappen nur 10 Prozent der Einwohner aus. Die meisten Einwohner der Siedlung arbeiten im Rentierzucht-Sowchos „Pamjat' Lenina”.

Ein wichtiger Faktor bei der Entwicklung der ethnokulturellen Prozesse sind Kontakte zu anderen Völkerschaften, die bei den Lappen sowohl in Lovozero als auch in Krasnoščel'e ein hohes Niveau erreichen. Lappen haben Umgang mit Vertretern anderer Nationalitäten auf der Straße, bei der Arbeit und in der Familie. Der Anteil der Mischehen beträgt bei den Lappen von Lovozero mehr als 50 Prozent, und zwar in fast 60 Prozent der Fälle mit einem russischen Ehepartner und zu einem Viertel mit einem 
Vertreter bzw. einer Vertreterin der Syrjänen.

Der Anteil der Mischehen bei den Lappen von Krasnoščele ist mit 57 Prozent noch größer. Etwa die Hälfte davon sind Ehen mit russischen Ehepartnern und ca. 40 Prozent mit Ehepartnern aus dem Kreis der Syrjänen.

Die Zahl der Mischehen bei den Lappen hat in den letzten 15 bis 17 Jahren stark zugenommen. Noch 1968 betrug der Anteil der Mischehen bei den Lappen von Lovozero, wie unsere Feldangaben jener Zeit besagen, lediglich 28 Prozent. Die zunehmende Zahl der Mischehen bei den Lappen ist zum einen auf das stetig anwachsende Niveau ihrer Kontakte zu anderen Völkerschaften, zum anderen aber, insbesondere in den letzten Jahren, auf die Unlust lappischer Mädchen, junge Männer ihrer eigenen Nationalität zu heiraten, zurückzuführen. Die Mädchen erklären das mit dem verschlossenen Charakter der jungen Lappen und mit ihrem Drang zu längeren Aufenthalten in der Tundra, wogegen die Mädchen am liebsten das ganze Jahr über in der Siedlung leben möchten, um in komfortablen Häusern wohnen und alle anderen Annehmlichkeiten eines größeren Ortes in Anspruch nehmen zu können. Es gibt vielleicht auch andere Gründe.

Die in letzter Zeit bei den Lappen festgestellte Tendenz, die in der rasch anwachsenden Zahl der Mischehen zum Ausdruck kommt, kann zu einer Bedrohung für die Existenz dieser von anderen Nationen umgebenen kleinen Völkerschaft ausarten. Unter diesen Bedingungen ist es sehr wichtig, wie die Kinder erzogen werden, welche ethnischen Werte sie von ihren Eltern mit auf den Weg bekommen, auf welcher Grundlage ihr ethnisches Selbstbewußtsein formiert wird und schließlich auch, für welche Nationalität sie sich entscheiden, wenn sie volljährig werden. An dieser Stelle sei gesagt, daß Kinder aus Mischehen in Lovozero, in denen einer der beiden Ehepartner Lappe ist, sich meistens für die lappische Nationalität entscheiden. Dazu tragen mehrere Faktoren bei. Erstens gehört in solchen Ehen die Frau meistens den Lappen, der Mann aber den Russen oder einer anderen Nationalität an. Da der mütterliche Einfluß in den ersten Lebensetappen des Kindes, wenn sein Weltgefühl sich herauszubilden beginnt und 
das Fundament für seine spätere ethnische Orientierung gelegt wird, des öfteren stärker ist als der des Vaters, so ist es nur natürlich, daß die Mutter im tagtäglichen Umgang mit dem Kind ihm, vielleicht nicht immer bewußt, die geistigen Werte ihrer eigenen nationalen Kultur, darunter auch die Muttersprache (falls sie diese beherrscht) vermittelt, die dann das erwachende ethnische Selbstgefühl des Kindes maßgeblich prägen.

Zweitens kann die Entscheidung der Kinder für die lappische Nationalität auch von ökonomischen Faktoren beeinflußt werden, gewährt ja der Staat den Kleinvölkerschaften des Hohen Nordens, zu denen auch die Lappen zählen, eine ganze Reihe von Vergünstigungen: kostenloser Aufenthalt der Kinder in Internatsschulen, bevorzugte Bedingungen bei der Aufnahme an höheren Bildungsstätten u. a. m. Und schließlich wird die Wahl der Nationalität bei den lappischen Kindern in letzter Zeit von einem Kulturprozeß bestimmt, der im Streben der Lappen nach Erhaltung und Weiterentwicklung der eigenen traditionellen Kultur zum Ausdruck kommt. Dieser Prozeß wird von Repräsentanten der lappischen Intelligenz, aber auch von den staatlichen Machtorganen gefördert. Das bezieht sich unter anderem auf die lappische Sprache, deren Erhaltung und Entwicklung gegenwärtig eine groBe Bedeutung beigemessen wird: Die Muttersprache der Lappen wird in der Schule erlernt, es liegen bereits eine Fibel und lappische Wörterbücher vor.

All das trägt selbstredend zur Erhaltung des nationalen Selbstbewußtseins bei der lappischen Bevölkerung bei, verstärkt ihr Interesse für die eigene Kultur und hat im Ergebnis zur Folge, daß die Zahl der Lappen nicht zurückgeht. Obwohl in den letzten 15 bis 17 Jahren der Prozentsatz der Mischehen bei den Lappen sich fast verdoppelt hat, ist die Gesamtzahl der Lappen in der UdSSR den beiden Volkszählungen von 1970 und 1979 nach zu urteilen praktisch unverändert geblieben: entsprechend 1884 und 1888 Menschen.

In der Siedlung Krasnoščele liegt eine etwas andere Situation vor. Angesichts eines vorherrschenden kulturellen Einflusses der Syrjänen entscheiden sich die Kinder aus Mischehen, wenn einer 
der beiden Ehepartner aus dem Kreis der Syrjänen, der andere aber aus dem Kreis der Lappen stammt, häufiger für die Nationalität der Syrjänen. Kinder aus Mischehen zwischen Lappen und Russen, Lappen und Nenzen sowie zwischen Lappen und Vertretern anderer Nationalitäten geben in Krasnoščel'e der Nationalität der Lappen in den meisten Fällen den Vorzug. Und das geschieht in einer Siedlung, wo die Anzahl der Lappen sehr gering ist und wo der Erhaltung ihrer nationalen Kultur keine so große Bedeutung beigemessen wird wie in Lovozero: In Krasnoščl'e wird zum Beispiel in der Schule kein Unterricht in Lappisch erteilt, und es wird nicht einmal eine entsprechende Forderung gestellt. Hier spielt möglicherweise der bereits erwähnte ökonomische Faktor eine Rolle. Es ist ferner nicht ausgeschlossen, daß hier eine überdurchschnittliche ethnische Stabilität der Lappen zum Ausdruck kommt, die es einer kleinen und bereits seit vielen Jahren in einer zahlenmäßig stark überlegenen anderssprachigen Umgebung lebenden Völkerschaft ermöglicht, ihr nationales Selbstbewußtsein zu erhalten.

Das hohe Niveau der Kontakte der Lappen zu anderen Nationen und der große Anteil der Mischehen bei den Lappen führten zu starken Wandlungen in ihrem sprachlichen Leben.

Die verhältnismäßig starke Isoliertheit der einzelnen Gruppen der lappischen Bevölkerung voneinander war eine der Ursachen für ihr sprachliches Getrenntsein voneinander. Die lappische Sprache der Lappen der Halbinsel Kola zerfällt in vier Dialekte, die nach den Orten Kildin, Jokanga, Notozero und Babino bezeichnet werden. Die Unterschiede zwischen den einzelnen Dialekten sind so groß, daß Lappen selbst einander manchmal nur mit Mühe und Not verstehen können. Innerhalb der Dialekte bestehen zahlreiche Lokalmundarten. Der Kildiner Dialekt schließt folgende Lokalmundarten ein: die Lovozeroer (Dorf Lovozero und Siedlung Teriberka), die Voron'eer (Dorf Voron'e), die eigentliche Kildiner (Dorf Čudsjavr) und die Semiostrov'eer (Dorf Varzino). Der Jokangaer Dialekt zerfällt in die Lokalmundarten der Dörfer Tuloma, Vosmus und anderer, die im Nordwesten der Halbinsel Kola liegen. Der Babinoer Dialekt repräsentiert die Lokalmund- 
arten der südwestlichen Gebiete der Halbinsel Kola. Die meisten Lappen, die den Kildiner und den Jokangaer Dialekt sprechen, leben gegenwärtig in Lovozero, wobei die Unterschiede zwischen diesen beiden Dialekten und den entsprechenden Mundarten innerhalb derselben erhalten bleiben. Die Lappen der Notozeroer Dialektgruppe, die vornehmlich im Dorf Tuloma mit einer national gemischten Bevölkerung leben und in verschiedenen für sie nichttraditionellen Wirtschaftsbereichen beschäftigt sind, haben ihre eigene Muttersprache praktisch eingebüßt und sprechen jetzt nur noch russisch. Die Lappen der Babinoer Dialektgruppe, die vornehmlich im Einzugsgebiet des Ënaer Dorfsowjets angesiedelt und ebenfalls in nichttraditionellen Wirtschaftsbereichen wie Milchviehhaltung und Ackerbau beschäftigt sind, sprechen auch fast alle nur noch russisch.

Unsere Untersuchungen der Lappen im Bezirk Lovozero haben ergeben, daß über 80 Prozent von ihnen die Muttersprache beherrschen, daß allerdings nur ein Drittel von ihnen neben der eigenen auch mehrere andere Lokalmundarten verstehen. Alle übrigen Befragten meinten, daß sie entweder nur ihre eigene Mundart oder höchstens nur noch eine benachbarte verstehen. Die erhalten gebliebenen großen Unterschiede zwischen den einzelnen Dialekten und Mundarten erschweren außerordentlich die Schaffung einer Schriftsprache der Lappen und einen entsprechenden Schulunterricht. Gegenwärtig wird Lappisch in der Vorschule und in den ersten Klassen unterrichtet. Im Kildiner Dialekt ist eine Fibel publiziert worden und sind ein russisch-lappisches und lappischrussische Wörterbücher erschienen. Die Arbeit an der Entwicklung einer Schriftsprache der Lappen geht weiter.

Lappisch findet neben der russischen und der syrjänische Sprache in verschiedenen Lebenssphären Gebrauch. Am meisten wird Lappisch in Familien, und dann speziell in mononationalen gesprochen. Fast die Hälfte der Lappen spricht in solchen Familien lappisch und russisch. Russisch hat aber für die Lappen weitaus breitere Funktionen. Russisch wird mit Arbeitskollegen und in Versammlungen gesprochen. Man liest russische Zeitungen und Zeitschriften, russische Belletristik, hört Rundfunk- und sieht 
Fernsehsendungen in Russisch. Die russische Sprache dient der Kommunikation zwischen den Angehörigen verschiedener Dialektgruppen. Auf diese Weise ist Russisch für die Lappen eine Sprache, die ihnen Kontakte sowohl zu anderen Nationen als auch innerhalb der eigenen Völkerschaft ermöglicht. Alle von uns befragten Lappen des Bezirks Lovozero sprechen fließend russisch. Gleiches gilt praktisch für alle Lappen im Gebiet Murmansk, wenn man das in Betracht zieht, was wir von den Lappen der Notozeroer und der Babinoer Dialektgruppe bereits gesagt haben. Die meisten von ihnen haben ihre Mittel-, eventuell auch ihre Fach- oder Hochschulbildung in Russisch genossen. Und doch ist das Interesse der Lappen für ihre Nationalsprache groß. Die Frage, ob sie möchten, daß ihre Kinder in der Schule Lappisch lernen, wurde von ca. 80 Prozent der Befragten, die Kinder im Vorschulalter hatten, positiv beantwortet. Das Interesse für die eigene Nationalsprache ist eine Erscheinungsform des ethnischen Selbstbewußtseins.

Die bei den Lappen der Halbinsel Kola entstandene Sprachsituation findet einen gewissen Niederschlag in ihren Angaben zur Muttersprache, die in den Ergebnissen der Volkszählungen fixiert wurden. Im Jahre 1959 betrachteten fast 70 Prozent der Kola-Lappen Lappisch als ihre Muttersprache, während es 1979 nur noch 53 Prozent waren. Für viele Lappen ist Russisch zur Muttersprache geworden, wobei 50 Prozent der Lappen sie für eine Zweitsprache halten, die sie voll beherrschen. Bemerkenswert ist aber die Tatsache, daß ein Teil der jungen Lappen, die nur schlecht lappisch sprechen, diese Sprache trotzdem als ihre Muttersprache betrachten. In diesem Fall tritt die Sprache als ethnisches Symbol in Erscheinung, als Mittel zur eigenen Identifizierung mit der ethnischen Gemeinschaft.

Der Wechsel der Muttersprache bei einem Teil der Bevölkerung, die Unterschiede in den Dialekten, ein hohes Niveau der Kontakte $\mathrm{zu}$ anderen Nationen und die territoriale Versprengung der Lappen scheinen die Assimilierungsprozesse unter den Lappen zu fördern. Die Ergebnisse der Volkszählungen stellen jedoch überzeugend unter Beweis, daß ihre Zahl nicht zurückgeht, was 
wiederum auf ein stabiles ethnisches Selbstgefühl der Lappen schließen läßt.

Abschließend läßt sich folgendes feststellen. Unsere Forschungen haben ergeben, daß die Lappen des Bezirks Lovozero im Gebiet Murmansk nach Charakter und Ausrichtung der ethnokulturellen Prozesse in zwei Untergruppen zerfallen: in die Lappen von Lovozero (der größere Teil) und die von Krasnoščel'e (der weitaus kleinere Teil). Lovozero ist eine Siedlung, wo die überwiegende Mehrzahl der im Gebiet Murmansk lebenden Lappen konzentriert ist und wo der Erhaltung ihrer Kultur, und insbesondere ihrer Sprache, ihrer Lieder, ihrer angewandten Kunst und ihrer Traditionen bedeutend mehr Aufmerksamkeit geschenkt wird als in Krasnoščl'e. Die nur mit 10 Prozent vertretenen Lappen von Krasnoščel'e entwickeln sich dort unter dem vorherrschenden Einfluß der Kultur der Syrjänen. 